\title{
Linear projection of technical noise for interferometric gravitational-wave detectors
}

\author{
J R Smith ${ }^{1}$, P Ajith ${ }^{1}$, H Grote ${ }^{1}$, M Hewitson ${ }^{1}$, S Hild ${ }^{1}$, H Lück ${ }^{1}$, \\ K A Strain ${ }^{2}$, B Willke ${ }^{1}$, J Hough ${ }^{2}$ and K Danzmann ${ }^{1}$ \\ ${ }^{1}$ Max-Planck-Institute for Gravitational Physics (Albert-Einstein-Institute) and \\ University of Hannover, Callinstr. 38, D-30167 Hannover \\ 2 Institute for Gravitational Research, Department of Physics and Astronomy, \\ University of Glasgow, Glasgow G12 8QQ, UK \\ E-mail: joshua.smith@aei.mpg.de
}

Received 26 September 2005, in final form 1 November 2005

Published 30 December 2005

Online at stacks.iop.org/CQG/23/527

\begin{abstract}
An international network of interferometric gravitational-wave detectors is now in operation, and has entered a period of intense commissioning focused on bringing the instruments to their theoretical sensitivity limits. To expedite this process, noise analysis techniques have been developed by the groups associated with each instrument. We present methods of noise analysis that were developed and utilized for the commissioning of the GEO 600 detector. The focal point of this paper is a technique called noise projection that is used to determine the levels of contribution of various noise sources to the detector output. Example applications of this method to control loops typical of those employed in an interferometric GW detector are presented. Possible extensions of noise projections, including technical noise subtraction and gravitationalwave vetoes are also discussed.
\end{abstract}

PACS numbers: $04.80 . \mathrm{Nn}, 95.55 . \mathrm{Ym}$

(Some figures in this article are in colour only in the electronic version)

\section{Introduction}

Throughout the world a number of long-baseline interferometers have been built, forming the most sensitive network of gravitational-wave $(\mathrm{GW})$ detectors ever. These consist of the three interferometers of the US-based LIGO [3], and one detector each for the Italian-French VIRGO [2], Japanese TAMA [1] and the German-British GEO 600 [4]. Teams of scientists within each group are currently in a state of intense work, known as commissioning, required to maximize the utility of the detectors. This is essentially divided into two parts. The first 
is to bring the detectors to robust states, allowing them to operate stably over long periods of time (many months). The second, often referred to as noise hunting, involves identification and elimination of any technical noise that couples significantly to the detector outputs. To help accomplish this, noise analysis techniques have been developed that allow estimation of the levels with which various technical noises couple into the detector output signals [5-7]. This paper describes the technique most often used to do this at GEO 600, which is called noise projection.

The following section introduces the technique of noise projections and presents a simplified procedure. Section 3 will provide example applications of this to control loops, highlighting the utility and limitations of the method. Section 4 will present possible ways to automate noise projections, and other extensions to the method.

\section{Noise projections}

To illustrate the scope of noise hunting related to commissioning an interferometric GW detector, we consider the characteristics of GEO 600. This detector is configured as a long-baseline $(600 \mathrm{~m})$ dual-recycled Michelson interferometer with folded arms (for more information about the optical layout, theoretical sensitivity limit, and main subsystems, see [4]). It is designed to detect strain signals with amplitudes on the order of $h \sim 1 \times 10^{-21}$. To achieve this, precise control systems are needed to keep the interferometer and all of its subsystems (laser frequency and power, beam alignment, etc) at their nominal operating points. For this reason, more than 200 control loops are associated with the operation of GEO 600 . Each of these can, in principle, couple noise to the detector output, reducing its sensitivity to gravitational waves. In addition, external disturbances, such as seismic or acoustic noise, can couple into the system through a variety of paths. Thus, the number of different noise sources that can contribute to limiting the detector sensitivity and the number of paths through which they may couple is very large.

In order to expedite commissioning, it is crucial to determine which noise sources contribute most to limiting the detector sensitivity such that the attention of the commissioners can be focused. While intuition and simple experiments are often sufficient to achieve this for projects of smaller scale, experience with commissioning at GEO 600 has shown that it is invaluable to systematically determine and track the level with which the various technical noises couple. The following subsection describes the noise projection procedure that is used for this purpose. In this paper, we assume that the noises couple linearly. For nonlinear noise couplings, a more sophisticated approach is needed.

Throughout this paper, we will refer to measurement points for time-domain signals as channels, and assume that they are continuously recorded using a data acquisition system like that used in GEO 600 [8]. Channels that are nominally intended to record signals that represent a given noise source, $n$, will be referred to as $N$, while the channel containing the detector output signal will be referred to as $E .^{3}$ The corresponding signals recorded by these channels are denoted with explicit time dependency, i.e. $N(t)$ and $E(t)$. A key point for the following discussion is that measurements are not always possible at every point within a system. We assume that measurements are only possible through channels, although we will sometimes refer to unmeasurable signals such as $n(t)$ to allow comparison.

3 We choose $E$, since the main detector output for interferometric gravitational-wave detectors is often a so-called error-signal that represents deviations in the Michelson interferometer differential arm-length degree of freedom from its operating point. 


\subsection{Procedure}

A simplified procedure for projecting a noise, $n$, that is recorded in channel $N$ to the detector output, $E$, is as follows:

(i) Establish the coupling of $n$ to $E$ by measuring the transfer function from $N$ to $E .^{4}$ This can be measured by taking the complex ratio

$$
T_{N \rightarrow E}(f)=\frac{\tilde{E}(f) \tilde{N}^{*}(f)}{\tilde{N}(f) \tilde{N}^{*}(f)},
$$

where $\tilde{N}(f)$ and $\tilde{E}(f)$ are complex Fourier transforms of $N(t)$ and $E(t)$, respectively, and $*$ denotes a complex conjugation. The transfer function should be determined while injecting a signal into the signal path before $N$ (nominally through the same path that $n(t)$ travels) that dominates the noise already present at $N$ and $E$.

(ii) Form spectra of the two signals during nominal conditions (without signal injected). The normalization of these spectra is inconsequential, but should be the same for both, since the goal of noise projections is a relative comparison. We use amplitude spectral density (ASD) since it is the most common normalization used by experimentalists to quantify noise levels, and denote the spectra of $N(t)$ and $E(t)$ as $N(f)$ and $E(f)$, respectively.

(iii) Multiply the spectrum of the noise channel and the absolute value of the measured transfer function $^{5}$. The resulting noise projection,

$$
P_{N \rightarrow E}(f)=N(f)\left|T_{N \rightarrow E}(f)\right|,
$$

is an ASD with units of equivalent $E(f)$ ASD. Comparison of the relative levels of $E(f)$ and $P_{N \rightarrow E}(f)$ gives the level with which the noise present in $N(f)$ couples to $E(f)$.

In order to apply this procedure to signals associated with control loops, it is necessary to take the characteristics of the loops into account. In section 3, we will demonstrate this through application of the method to example control loops.

First we will discuss a few of the variants of the noise projection technique. In the following we use the terms in-loop and out-of-loop to describe measurement points that contain signals that are within, or external to, the causal signal flow of a given control loop, respectively. The state of control loops that are either nominally operating, or that have a break in signal flow at some point, is referred to as closed loop and open loop, respectively.

\subsection{In-loop projections}

Noise levels and couplings may depend on the state of the entire system, and since most control loops associated with GEO 600 are closed in order to keep the detector in this state, it is desirable to project the in-loop signals of these closed loops. However, within a feedback loop, the mixing of noises due to loop gain sets a limit on the utility of in-loop measurements. In the next section we demonstrate that for single-path loops, noise projections can only be interpreted with certainty for frequencies at which the loop gain of the loop containing $N$ is less than untiy. Although this imposes a severe limit on the utility of this method within control loops whose unity gain frequency (UGF) is high compared to the detection band of the instrument (typically 50-2000 Hz), it should be noted that many of the control loops

4 Alternatively, the transfer function can be calculated using a model. We have found that measuring the transfer functions while the system is in its nominal state is less prone to errors, since it does not depend, as does a model, on the accuracy with which parameters of the system are known. It is a good idea to compare the measured transfer function with that predicted by a model to check that the coupling is understood, and to refine the model.

5 This is equivalent to multiplying the complex quantities $\tilde{N}(f)$ and $T_{N \rightarrow E}(f)$ and then normalizing to ASD, since $|A B|=|A||B|$. Since we seek a comparison with ASD, we neglect the phase information. 
in gravitational-wave detectors are employed to reduce deviations (for example, seismically driven mirror motions) that occur below the detection band, and thus have loop gain less than unity over this band.

\subsection{Out-of-loop projections}

Channels that measure useful out-of-loop signals while the detector is in its nominal state are ideal for noise projections since they can be measured continuously without the loop-gain limitations mentioned above. These channels exist where there is a reference that measures the deviations of a given parameter independent of a control loop (e.g. a fixed reference cavity to independently measure frequency deviations of the laser at low Fourier frequencies). However, it should be noted that during the course of commissioning, once such a channel has been shown to measure a noise that couples significantly to the detector output, it is often converted (by the commissioners) into an error signal and fed back to reduce the coupling. Thus such channels may exist only transiently.

\subsection{Open-loop projections}

If no out-of-loop measurements of the type described above exist for a given noise, and if in-loop measurements do not give sufficient information due to loop-gain limitations, it may still be possible to determine the level of noise coupling by making the ASD measurements on an open loop (the transfer functions are still measured in-loop). Many noise sources within a given control loop can be isolated by breaking the signal flow at different locations, such that only the desired noise adds to the measured channel. In the open loop, the measured signals are not influenced by loop gain. Thus it is necessary to multiply the ASDs by the closed-loop transfer function of the loop containing $N$ in order to project the correct level of noise coupling. The drawback of this type of measurement is that the entire system is not at its nominal state, and thus various noises that depend on the state of the system may appear at different levels, or not at all.

\section{Example applications}

In this section we present examples to illustrate how noise projections work when applied to realistic control loops. The examples given are not intended to be exhaustive or general, but rather indicative of the main features and drawbacks of the method. Since the application of out-of-loop noise projections is straightforward, following the procedure described in the last section, we focus on in-loop noise projections and the limits imposed on them by loop gain. Although a variety of control techniques exist, (e.g. both feedback and feedforward [9] control are used within GEO 600), we consider only the most commonly used control type at GEO 600, linear inverting feedback. This works by measuring the deviation of a parameter of a given system (the plant) from a desired operating point. The result, called the error signal, is passed through filters (the servo) that transform it into a feedback signal of opposite sign. This is fed back to the system, reducing the deviation from the operating point by the closed-loop transfer function of the loop (in the steady state). In the following, since we are only concerned about signals that are measurable, we will consider the plant, $G$, to also include the device that senses the deviations from the operating point (the sensor) and that which actuates with the correction signal (the actuator). 


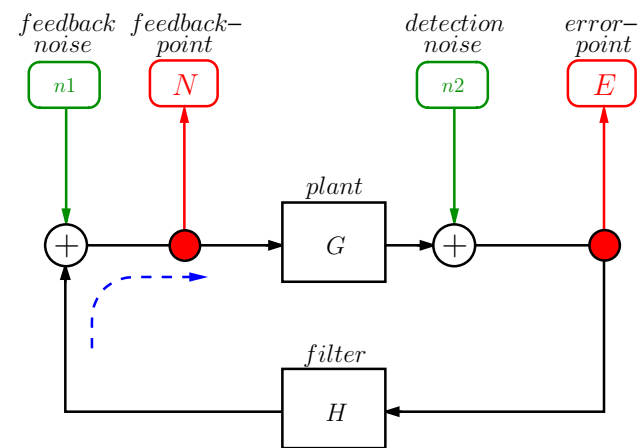

Figure 1. Single-path feedback loop consisting of a plant and filter, two independent noise sources and two measurement channels. Signals flow clockwise through the loop, as indicated by the dashed arrow.

\subsection{Single loop}

As a first example, we consider the single-path feedback loop shown in figure 1. This consists of a plant, $G$, and a filter, $H$, and has two independent noise sources, $n_{1}$ and $n_{2}$ (suggestively referred to as feedback noise and detection noise because of their locations). The channels, $E$ and $N$, represent the error point and feedback point of the loop, respectively. We are interested in determining the level with which $n_{1}$, a noise added in the actuation chain, couples to $E$ by performing the in-loop noise projection $P_{N \rightarrow E}$.

For the following, it is useful to know the open-loop gain, $G_{\mathrm{OL}}$, and closed-loop transfer function, $T_{\mathrm{CL}}$, of the loop,

$$
G_{\mathrm{OL}}=G H, \quad T_{\mathrm{CL}}=\frac{1}{1+G_{\mathrm{OL}}} .
$$

When the loop is closed, $N(t)$ and $E(t)$ will contain a combination of the noises $n_{1}$ and $n_{2}$

$$
N=\left(n_{1}+n_{2} H\right) T_{\mathrm{CL}} \quad E=\left(n_{1} G+n_{2}\right) T_{\mathrm{CL}} .
$$

Measurement of the transfer function, $T_{N \rightarrow E}$ (performed in-loop, while injecting a dominant signal through the path that $n_{1}$ takes), yields $G$.

The levels with which $n_{1}(t)$ and $n_{2}(t)$ couple to $E(t)$ can be determined accurately by breaking the signal flow in such a way that $n_{1}(t)$ and $n_{2}(t)$ are isolated into channels $N$ and $E$, respectively, for the ASD measurement, and making open-loop noise projections,

$$
\begin{aligned}
& P_{n_{1} \rightarrow E}=\left.N(f)\right|_{n_{1}} T_{\mathrm{CL}} T_{N \rightarrow E}=n_{1}(f) T_{\mathrm{CL}} G, \\
& P_{n_{2} \rightarrow E}=\left.E(f)\right|_{n_{2}} T_{\mathrm{CL}} T_{E \rightarrow E}=n_{2}(f) T_{\mathrm{CL}},
\end{aligned}
$$

where $\left.\right|_{n_{x}}$ indicates that the signal is dominated by noise $n_{x}$. Note that both projections include $T_{\mathrm{CL}}$, since the ASDs were measured in the open loop. These can be used as references with which to judge the validity of the in-loop projection.

The in-loop projection of $N(t)$ to $E(t)$ is

$$
P_{N \rightarrow E}=N(f) T_{N \rightarrow E}=\left(n_{1}+n_{2} H\right) T_{\mathrm{CL}} G .
$$

It can be shown that $P_{N \rightarrow E}$ gives the correct level of noise at $E$ for any noise added after $E$ and before $N$ (such as our feedback noise, $n_{1}$ ), as long as it dominates $N(t)$. If a noise added after $N$ and before $E$ (such as our detection noise, $n_{2}$ ) dominates $N(t)$, the apparent projection 

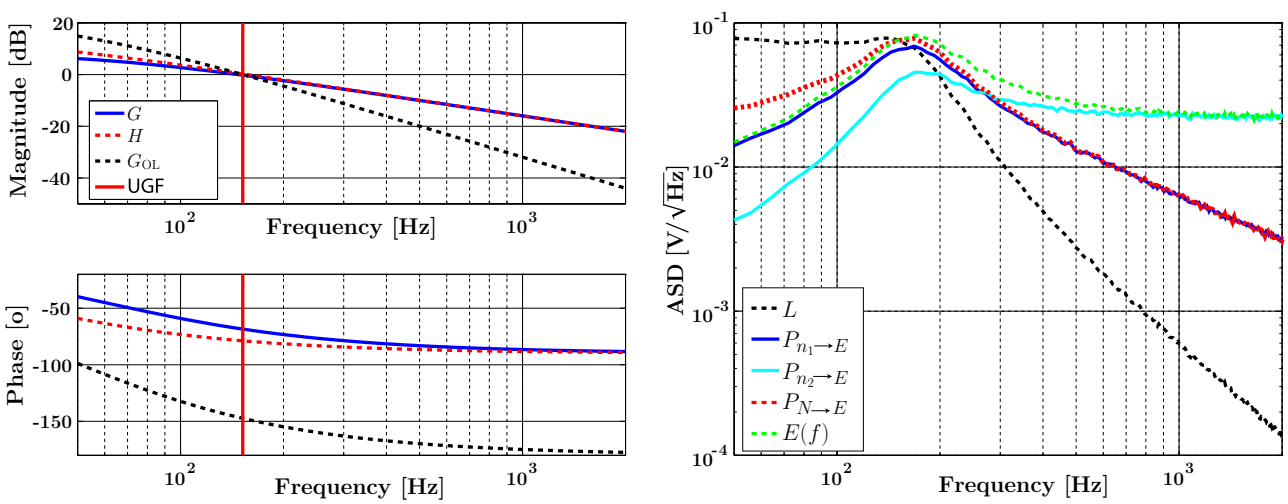

Figure 2. Results of simulation of a single-path feedback loop. Left: $G, H$, and the open-loop gain of the entire loop. Right: amplitude spectral densities of the noise projections, the detector output and the loop-noise upper limit.

(recall that we seek to project $n_{1}$ ) will equal $E(f) G_{\mathrm{OL}}$. Thus, $E(f) G_{\mathrm{OL}}$ is an upper limit on the error of the in-loop projection that is caused by noise added between points $N$ and $E$. We refer to this as the loop-noise upper limit, or $L$, and its normalization (by $E(f)$ ) as $\hat{L}$. For frequencies over which the latter is greater than untiy, definite information about the level of coupling of a given noise to the detector output cannot be obtained using in-loop noise projections.

Typical control loops have frequency-dependent loop gain and noise contributions from several noise sources. It is thus useful to see how well noise projections perform when applied to an example loop with these characteristics. For this we consider the loop in figure 1, and make $G$ and $H$ frequency-dependent gains and $n_{1}$ and $n_{2}$ uncorrelated random noises with similar variances. Responses of $G, H$ and the open-loop gain of the full loop are shown in the left graph of figure 2. The unity-gain frequency (UGF) is $140 \mathrm{~Hz}$.

The right graph of figure 2 shows the ASDs resulting from noise projections performed on a simulation of this loop. The actual couplings of $n_{1}$ and $n_{2}$ to $E$ are determined by the out-of-loop projections $P_{n_{1} \rightarrow E}$ and $P_{n_{2} \rightarrow E}$. In addition, an in-loop projection of $P_{N \rightarrow E}$ is performed. By comparing these, we can determine how accurately the in-loop projection represents the coupling of $n_{1}$ into $E(t)$. Above the UGF, $P_{N \rightarrow E}$ agrees well with $P_{n_{1} \rightarrow E}$, and is well above the loop-gain upper limit (which gives confidence of the validity of the projection even without the out-of-loop projection for comparison). Below the UGF, the projection is an overestimate of the actual contribution due to contamination of $N(t)$ by $n_{2}$.

To summarize, within a single loop, an in-loop projection from $N$ to $E$ is valid for frequencies at which a noise added after $E$ and before $N$ dominates $N(t)$. For frequencies at which this is not true, the projection can overestimate the noise coupling by the loop-noise upper limit set by $G_{\mathrm{OL}} E(f)$. Dependable information can be gleaned only at frequencies above the UGF and for which the projection is above $L$. If these conditions are not met for the noise source and frequencies of interest, the ASDs must be measured with the loop open.

\subsection{Split-path loop}

Next we consider the split-path loop shown in figure 3. This consists of a plant, $G$, and two filters, $H_{1}$ and $H_{2}$, which are in separate paths called the slow and fast path, respectively. Independent noise sources are added at $n_{1}, n_{2}$ and $n_{3}$ and measurements are made through the 


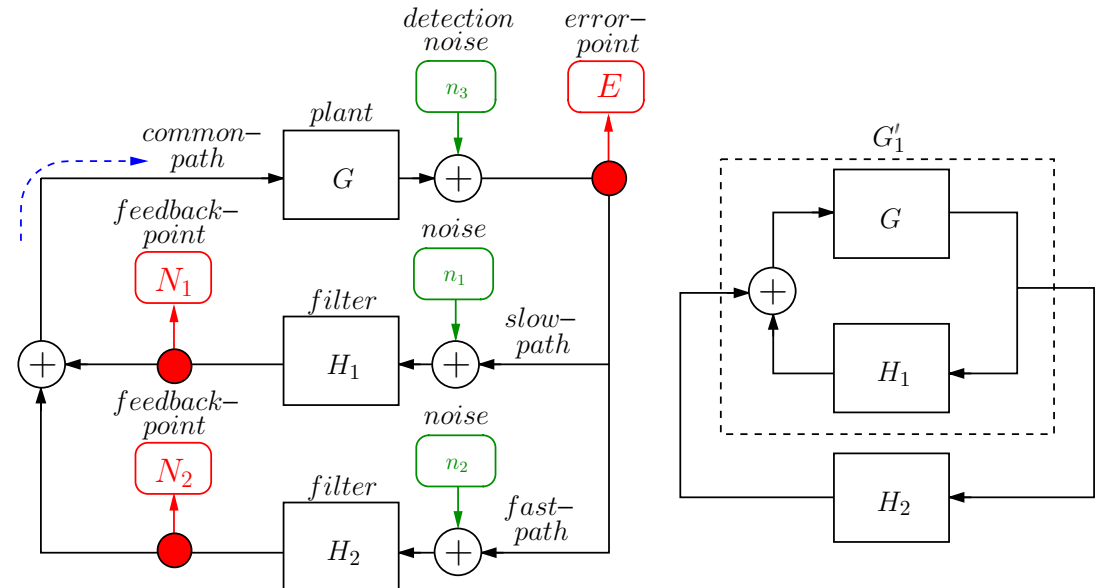

Figure 3. Left: split-path feedback loop consisting of a plant and two filters, each on a separate path. Three independent noise signals are added and signals are recorded by three measurement channels. Signals flow clockwise, as indicated by the dashed arrow. Right: a rearrangement of the loop as a single-path loop consisting of the equivalent gain of the slow path and the filter for the fast path.

channels $N_{1}, N_{2}$ and $E$. We are interested in projecting the level of the coupling of the noises added in the two individual paths to $E(f)$. Although the following equations are general, we point out that in a typical split-path loop, the slow path has higher gain than the fast path at low frequency and lower gain at high frequency, and the frequency at which the gains of the two paths are equal is called the crossover frequency and is lower than the unity-gain frequency.

The open-loop gain of the entire loop and the slow and fast paths are

$$
G_{\mathrm{OL}}=G\left(H_{1}+H_{2}\right), \quad G_{\mathrm{OL} 1}=G H_{1} \quad \text { and } \quad G_{\mathrm{OL} 2}=G H_{2} .
$$

The closed-loop transfer function of the entire loop (measured in the common path) is

$$
T_{\mathrm{CL}}=\frac{1}{1+G_{\mathrm{OL}}} .
$$

In order to calculate the closed-loop transfer functions of the separate paths (i.e. the amount of suppression a signal injected into one of these paths experiences at the point of injection), it is useful to form an equivalent single-path loop through simplification, as shown in the right diagram of figure 3 . Here the slow path and the plant form an equivalent gain,

$$
G_{1}^{\prime}=\frac{G}{1+G H_{1}} .
$$

Similarly for the fast path we get

$$
G_{2}^{\prime}=\frac{G}{1+G H_{2}} .
$$

It follows that the closed-loop transfer functions for the two split paths are

$$
\begin{aligned}
& T_{\mathrm{CL} 1}=\frac{1}{1+G_{2}^{\prime} H_{1}}=\frac{T_{\mathrm{CL}}}{1+G H_{2}} \\
& T_{\mathrm{CL} 2}=\frac{1}{1+G_{1}^{\prime} H_{2}}=\frac{T_{\mathrm{CL}}}{1+G H_{1}} .
\end{aligned}
$$



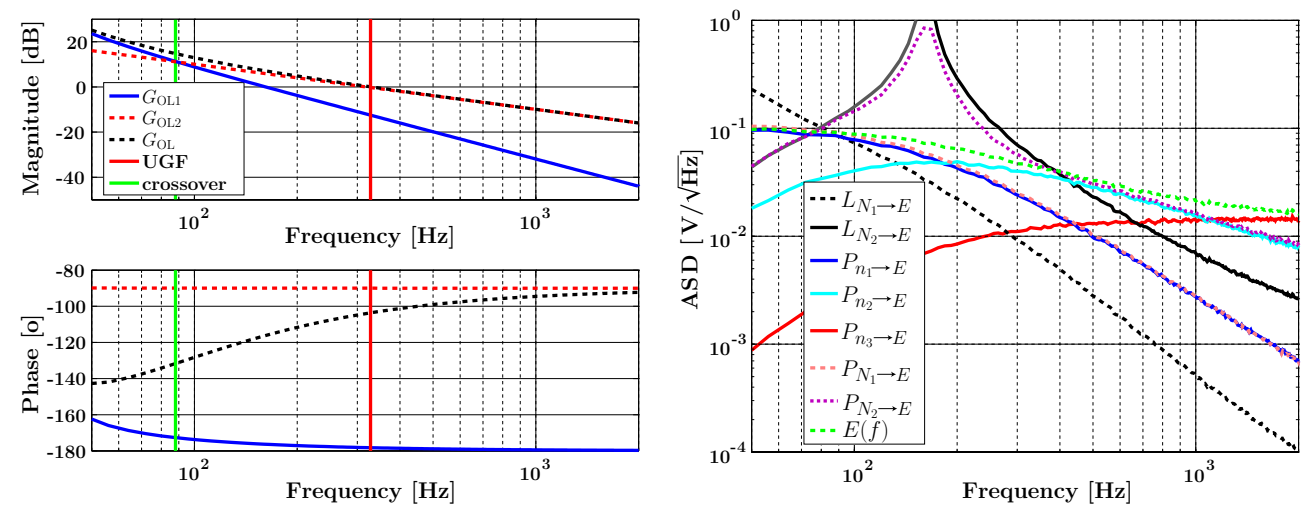

Figure 4. Results of simulation of a split-path feedback loop. Left: open loop gain of the slow and fast paths, and full loop. Right: amplitude spectral densities of the noise projections, the detector output and the loop-noise upper limits.

When the loop is closed, the signals that appear in the three channels are linear combinations of the three noise sources,

$$
\begin{aligned}
& E=n_{1} T_{\mathrm{CL} 1} H_{1} G_{2}^{\prime}+n_{2} T_{\mathrm{CL} 2} H_{2} G_{1}^{\prime}+n_{3} T_{\mathrm{CL}} \\
& N_{1}=n_{1} T_{\mathrm{CL} 1} H_{1}+n_{2} T_{\mathrm{CL} 2} H_{2} G_{1}^{\prime} H_{1}+n_{3} T_{\mathrm{CL}} H_{1} \\
& N_{2}=n_{1} T_{\mathrm{CL} 1} H_{1} G_{2}^{\prime} H_{2}+n_{2} T_{\mathrm{CL} 2} H_{2}+n_{3} T_{\mathrm{CL}} H_{2} .
\end{aligned}
$$

Measurement of the noise-coupling transfer functions for $N_{1}$ and $N_{2}$ yield

$$
T_{N_{1} \rightarrow E}=\left.\frac{E}{N_{1}}\right|_{n_{1}}=G_{2}^{\prime} \quad T_{N_{2} \rightarrow E}=\left.\frac{E}{N_{2}}\right|_{n_{2}}=G_{1}^{\prime} .
$$

The projections are found according to equation (2).

We can set upper limits on the error of in-loop projections of the two feedback-point channels by assuming their signals are dominated by noise added outside of the path in which they are situated (i.e. either in the common path or in the fast path if the channel is in the slow path). This gives normalized loop-noise upper limits,

$$
\begin{aligned}
& \hat{L}_{N_{1} \rightarrow E}=\left.\frac{E}{N_{1}}\right|_{n_{2}}=\left.\frac{E}{N_{1}}\right|_{n_{3}}=H_{1} G_{2}^{\prime}=\frac{G H_{1}}{1+G H_{2}}, \\
& \hat{L}_{N_{2} \rightarrow E}=\left.\frac{E}{N_{2}}\right|_{n_{1}}=\left.\frac{E}{N_{2}}\right|_{n_{3}}=H_{2} G_{1}^{\prime}=\frac{G H_{2}}{1+G H_{1}} .
\end{aligned}
$$

It can be shown that these upper limits hold for the case where $N_{x}$ is not dominated by a noise added in the $x$-path. Split-path loops typically have high loop gain at the crossover frequency and much more gain in the fast path at the UGF than in the slow path. Applying these conditions to the above formulae shows that the normalized upper limits equal or exceed unity at and below the crossover frequency for the slow path, and at and below the UGF for the fast path ${ }^{6}$.

Figure 4 shows the results of a simulation for the control loop shown in figure 3 with uncorrelated random noises and frequency-dependent gains for each path. The open-loop gain of the slow and fast paths and the entire loop are shown on the left. The right graph shows the

6 Note however that below the crossover, where $G_{\mathrm{OL} 1}>G_{\mathrm{OL} 2}, \hat{L}_{N_{2} \rightarrow E}$ will again be less than 1 . 
results of the noise projections. As expected, the upper limits on the in-loop projections of the slow- and fast-path feedback points are equal to or greater than $E(f)$ below the crossover frequency $(88 \mathrm{~Hz})$ and unity-gain frequency $(310 \mathrm{~Hz})$, respectively. Above these frequencies the in-loop noise projections are accurate representations of the actual noise coupling.

To summarize, in-loop noise projections performed on signals within the two separate paths of a split-path feedback loop are subject to two different loop-noise upper limits. For a typical split-path loop, $L$ will be equal to or greater than $E(f)$ below the crossover frequency for the slow path, or the UGF for the fast path. Thus in split-path loops, in-loop noise projections of signals in the slow path can give valuable information even below the UGF.

\subsection{Coupled loops}

As discussed in section 2, noise entering a different control loop than that containing the detector output may couple, through a variety of paths, and contribute to $E$. Thus projection of noises that propagate through coupled loops is often needed. This can be done, as in the previous examples, following the procedure given in section 2. The normalized loop-noise upper limit is determined by the gain properties of the loop containing $N$, (e.g. it would equal the open-loop gain if $N$ were within a single-loop like that shown in figure 1). And the loopnoise upper limit would be given by multiplying this with the noise of the loop containing $N$ at the point where it exits the loop and enters the coupling path. Since this noise is often not measurable (if it were, it would be better to use it as an open-loop measurement channel for projecting), the normalized loop-noise upper limit can be multiplied by $E$, giving a higher, but still useful upper limit.

\section{Extensions of the technique}

\subsection{Automation}

Noise projections done at single points in time are sufficient to give commissioners the information they need to identify the most important noise sources at the time of measurement. However, since couplings and noise levels may drift over time, it is useful to have noise projections available at arbitrary times (e.g. during a data run) and even continuously. Since the noise ASDs can be measured continuously during nominal detector operation, automation requires only a small modification of the procedure given in section 2 , namely tracking the time evolution of noise couplings. It is not practical to measure a transfer function for each noise channel at all times following the procedure presented in section 2, because adding dominant signals to the system would reduce the sensitivity of the detector ${ }^{7}$. A possible technique for slowly varying couplings is to inject a dominant noise into the system for each noise coupling periodically for short amounts of time (e.g. $100 \mathrm{~s}$ per hour), and to use these measurements as the transfer functions for that period. Alternatively, the transfer functions can be determined continuously at certain frequencies using injected periodic signals. If a given noise coupling is known to vary primarily by an overall gain, a signal at a single frequency is sufficient to track its gain changes over time. For frequency-dependent variations, the transfer function can be probed using signals at several frequencies and then fit to a parametrized model as is done in the GEO 600 calibration process $[10,11]$.

\footnotetext{
7 It is theoretically possible to inject pseudo-random noise at all points and subtract it from the detector output using
} the measured transfer functions, but this is technically difficult and requires that the system behave quite linearly. 


\subsection{Technical-noise subtraction}

As described above, the output of noise projections is a spectrum in units of projected contribution to the detector output. Thus a natural extension is to subtract the projected noise from the detector output signal. To do this, it is necessary to carry out the noise projection in the time domain. This can be done by passing $N(t)$ through a time-domain filter that represents the measured noise transfer function over the frequency range of interest (i.e. where the projection is above the loop-noise upper limit and where noise subtraction can be beneficial). The resulting signal can be thought of as a feedforward actuation signal that can be subtracted from the detector output. The noise reduction possible with this system depends on the accuracy with which the filter represents the actual noise coupling. A factor of ten reduction requires that the filter be roughly within about $10 \%$ in amplitude and $5^{\circ}$ in phase (for a calculation of this, see [9]). Thus, to make time-domain noise subtraction practical, either verified long-term stability of the noise coupling or real-time adaptive monitoring are needed. This can be achieved using the methods described at the end of section 4.1. To avoid complicating the calibration of the detector output, it is necessary to ensure that GW signals do not couple significantly into the subtraction signal.

\subsection{Vetoes against false gravitational waves}

Noise projections can also be extended to help reduce the influence of technical noise on the analysis of the detector output data for gravitational-wave information, by vetoing candidate gravitational-wave events that are in fact detector artefacts. As an example, consider a search for gravitational-wave burst events that uses an event-trigger generator (ETG) to generate, from the detector output data, $E(t)$, a list of candidate events that are parametrized by their time of occurrence, central Fourier frequency, and amplitude. If we now run this same ETG on the noise channel signal, $N(t)$, and generate a likewise parametrized list of events, these events can be projected to the detector output using the transfer function from $N$ to $E$, giving a list of technical-noise events with the parameters with which they are expected to have in $E(t)$. Projected technical-noise events with parameters that match those of the detector output events (within errors) can be used as gravitational-wave vetoes. This is a subject of continuing research within our group.

\section{Summary}

We have discussed methods for performing noise projections, and have shown how they can be useful for determining the levels of coupling of noises through feedback control loops. A limit to the utility of in-loop noise projections is imposed by the mixing of noise from different sources due to loop gain. In order to quantify this, we defined the loop-noise upper limit, and showed that in-loop noise projections do not provide definite information for frequencies at which this is greater than the detector output (i.e. the normalization is greater than one). This occurs below the unity-gain frequency for single-path loops and the fast path of split-path loops, and below the crossover frequency for the slow path of split-path loops. Since most control loops in GEO 600 have unity-gain frequencies below $100 \mathrm{~Hz}$, in-loop noise projections can give valuable information in the gravitational-wave detection band $(\approx 50-2000 \mathrm{~Hz})$.

We have also discussed possible extensions of noise projections. Automation, through the tracking of the time evolution of noise-coupling transfer functions, can lead to a continuous knowledge of the coupling of technical noise to the detector output, an invaluable tool for detector commissioning. Reduction of the coupling of technical noise to the detector output 
can be achieved using a time-domain filter to approximate the noise-coupling transfer function, allowing noise subtraction. Finally, using the noise projection technique, it is possible to veto candidate events during the analysis of the detector output for gravitational-wave information.

The successful application of the procedures discussed in this paper to the technical noise of GEO 600 is the subject of another paper.

\section{Acknowledgments}

We thank M Malec for useful comments on the manuscript. We are grateful for support from PPARC and the University of Glasgow in the UK, and the BMBF and the state of Lower Saxony in Germany.

\section{References}

[1] Takahashi R et al 2004 Status of TAMA300 Class. Quantum Grav. 21 S403

[2] Frasconi F et al 2004 Status of VIRGO Class. Quantum Grav. 21 S385

[3] Sigg D et al 2004 Commissioning of LIGO detectors Class. Quantum Grav. 21 S409

[4] Willke B et al 2002 The GEO 600 gravitational wave detector Class. Quantum Grav. 191377

[5] Adhikari R 2004 Sensitivity and noise analysis of $4 \mathrm{~km}$ laser interfermetric gravitational wave antennae, PhD Thesis Massachusetts Institute of Technology (http://www.ligo.caltech.edu/docs/P/P040032-00.pdf)

[6] Flaminio R, Gouaty R and Tournefier E 2004 Search for Noise Sources in C1 and C2 Sensitivities internal document of VIRGO (http://wwwlapp.in2p3.fr/virgo/notes/Notes2004 /VIR-NOT-LAP-1390-277.pdf)

[7] Tatsumi D Private communication

[8] Kötter K et al 2002 Data acquisition and detector characterization of GEO 600 Class. Quantum Grav. 191399

[9] Smith J R, Grote H, Hewitson M, Hild S, Lück H, Parsons M, Strain K A and Willke B 2005 Feedforward correction of mirror misalignment fluctuations for the GEO 600 gravitational wave detector Class. Quantum Grav. 223093

[10] Hewitson M, Heinzel G, Smith J R, Strain K A and Ward H 2004 Principles of calibrating the dual-recycled GEO 600 Rev. Sci. Instrum. 754702

[11] Hewitson M et al 2004 Calibration of the dual-recycled GEO 600 detector for the S3 science run Class. Quantum Grav. $21 \mathrm{~S} 1711$ 